\title{
O ENSINO VOLTADO AS CONSTANTES DA FÍSICA, TAIS COMO GRAVIDADE LOCAL, CONSTANTE DA MOLA E COEFICIENTE DE DILATAÇÃO VOLUMÉTRICA
}

\section{THE BACKGROUND TEACHING THE CONSTANTS OF PHYSICS, SUCH AS LOCAL GRAVITY, CONSTANT OF THE SPRING AND COEFFICIENT OF VOLUMETRIC DILATION}

\author{
Nelson da Silva Nunes - UFAL/Brasil \\ José Renan Gomes dos Santos - UFAL/Brasil
}

\begin{abstract}
RESUMO: O objetivo desse artigo é abordar a importância das constantes físicas através de alguns experimentos simples o qual se remete a medição da constante elástica, a constante gravitacional e a dilatação volumétrica. O trabalho foi desenvolvido com seis alunos do terceiro ano do ensino médio, de uma escola publica estadual Maria Salete de Gusmão, onde o mesmo foi realizado em quatro encontros de cinquenta minutos. As atividades foram desenvolvidas com o intuito de levar os alunos a construírem significados físicos para algumas constantes. Os principais resultados encontrados no desenvolvimento desse projeto foi a percepção dos alunos que as constantes têm uma relevante importância de uma modelagem matemática, onde elas quebram as proporcionalidades apresentadas matematicamente. Portanto, podemos afirmar que o presente artigo mostra que é relevante para prática didática e formação do conhecimento, a fim de gerar um saber construtivo e sólido na vida escolar do aluno e na didática do professor de física.
\end{abstract}

Palavras-chave: Constantes Física. Experimentos. Ensino de Física.

ABSTRACT: The objective of this work is to discuss the importance of the physical constants through some simple experiments which refers to the measurement of the elastic constant, the gravitational constant and the volumetric expansion. The study was carried out with six students from the third year of high school at a state public school Maria Salete de Gusmão, where it was held in four fifty-minute meetings. The activities were developed with the purpose of getting students to construct physical meanings for some constants. The main results found in the development of this project were that the students were able to perceive that the constants have an important importance behind a mathematical modeling, where they break the proportions presented mathematically. Therefore, we can affirm that the present article shows that it is relevant for didactic practice and knowledge formation, in order to generate a constructive and solid knowledge in the school life of the student and in the didactics of the physics teacher..

Keywords: Constants Physics. Experiments. Physics Teaching.

\section{INTRODUÇÃO}

Educação, Psicologia e Interfaces, Volume 2, Número 2, p. 77-85, Maio/Agosto, 2018.

ISSN: 2594-5343. DOI: https://doi.org/10.37444/issn-2594-5343.v2i2.61 
Tradicionalmente, reconhecemos o conhecimento físico a partir do uso de expressões matemáticas que relacionam as variáveis (dependentes e independentes), no entanto, estas expressões são apresentadas aos alunos de forma pronta e acabadas, ou seja, a Lei da gravitação Universal de Newton, por exemplo, é simplesmente apresentada na

sua forma matemática, $F=\frac{M m}{d^{2}} G$, com pouca ou nenhuma discussão física sobre como se comportam as variáveis.

Para promovermos a discussão sobre o papel das constantes no Ensino de Física usaremos três experimentos cujo foco recai na determinação da constante, todavia se torna complexo a análise teórica desse tema devida a falta de referências bibliográficas que destrinchem esse assunto.

Os conhecimentos físicos são saberes que necessitam muitas vezes serem expressos por equações matemáticas, a essa representação matemática que construímos a partir da análise do comportamento dos fenômenos naturais, e das construções gráficas. Definimos modelos matemáticos com uma ideia de tornar mais simples o que é dito como real, a modificação do concreto é essencial para uma melhor compreensão de determinado aspecto científico a ser estudado pelos alunos, porem como cita (VASQUES, 2008, p.10) essa modificação da realidade pode ser um problema na construção do conhecimento do aluno, “...o mundo abstrato(ideal) e o mundo concreto (real). Esta desconexão entre teoria e realidade é um problema frequentemente observado...".

Algo importante de se observar nisso é que as equações que surgem das modelagens matemáticas, geralmente nos trazem constantes que nos fornecem de maneira mais eficiente o comportamento da natureza. No entanto, o problema não está em como conhecimento físico é construído, estruturado pela matemática, mas como ele é ensinado nas turmas de Ensino Médio. O objetivo deste trabalho é propor uma metodologia de aula para o professor trabalhar as constantes físicas em sala de aula para ir além da pura matematização dos fenômenos.

\section{METODOLOGIA}

Para atender ao objetivo do trabalho optamos por realizar uma pesquisa experimental envolvendo alunos do Ensino Médio. Nesse tipo de trabalho, procuramos controlar as variáveis relevantes para o desenvolvimento das atividades, tais como, 
O ensino voltado as constantes da física, tais como gravidade local, constante da mola e coeficiente de dilatação volumétrica

quantidade de aulas, uso ou não de atividades, o tipo de atividades, questionamentos e metodologias.

Os alunos que participaram desta pesquisa foram escolhidos de forma voluntária entre os alunos de uma turma do $3^{\circ}$ Ano do Ensino Médio de uma escola estadual, da periferia de Maceió - AL, integrando do PIBID/UFAL. Participaram seis alunos divididos em dois grupos com três.

\section{RESULTADOS E DISCUSSÃO}

Nesta seção iremos apresentar os três experimentos desenvolvidos para serem usados pelos alunos durante as aulas. Os experimentos que foram escolhidos para serem utilizados nesta pesquisa necessitavam a existência de constantes em seus modelos matemáticos.

O primeiro experimento utilizou massas aferidas em um dinamômetro (ver figura 1) onde os alunos deveriam possuir o conhecimento prévio de força peso para assim poderem identificar à constante, já no experimento de medição da constante da mola (ver figura 2), utilizamos novamente massas aferidas, um tripé e um suporte para aferir as massas em uma mola a qual sua constante elástica era desconhecida.

Figura 1 : Suporte experimental para calcular gravidade local

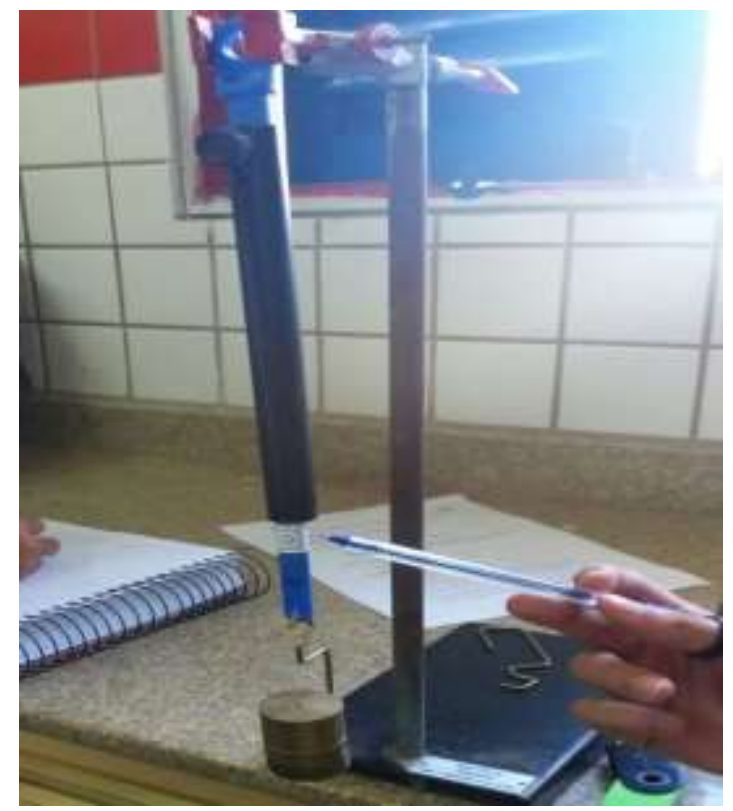

Fonte: Nelson S.Nunes, Maceió 06 junho de 2017.

Figura 2 : Suporte experimental para calcular a constante da mola 


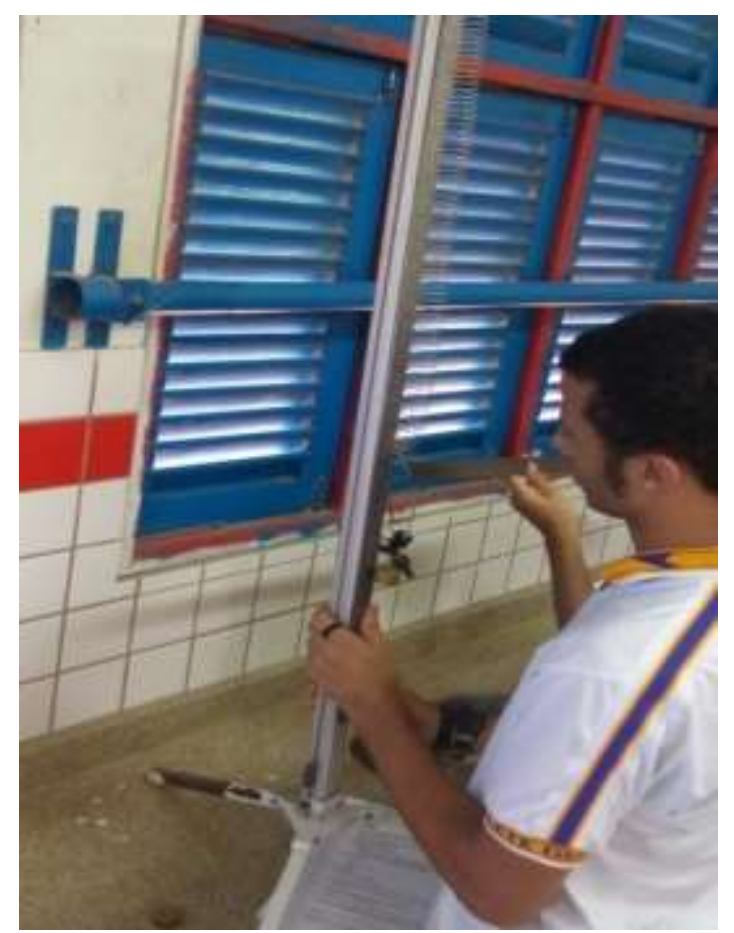

Fonte: Nelson S.Nunes, Maceió 13 junho de 2017.

No terceiro experimento (ver figura 3) utilizamos uma jarra de Becker onde aquecemos a água, utilizamos também uma pequena garrafa com uma seringa presa em sua ponta, onde a garrafa estava completamente vedada, observamos a dilatação, conforme íamos aquecendo a água contida no Becker o fluido contida na garrafa pet também ia se aquecendo pelo processo de transmissão de calor e consequentemente o liquido ia se dilatando.

Em seguida fizemos o mesmo procedimento porem utilizamos uma panela com água, gelo e sal, com a itenção de criamos uma mistura onde o seu ponto de fusão estivesse abaixo de zero para observar a contração, onde tínhamos uma seringa acoplada na garrafa e totalmente vedada a base de pressão e força, existia na mesma um fluido (água ), a medida que o tempo passava o volume de água contido na seringa diminuía de forma gradativa, pois existia uma troca de calor mútua entre o recipiente com água abaixo de zero e a garrafa pet para que se tornasse possível a visualização da contração térmica.

Figura 2: Suporte experimental para calcular o coeficiente de dilatação volumétrica. 
O ensino voltado as constantes da física, tais como gravidade local, constante da mola e coeficiente de dilatação volumétrica

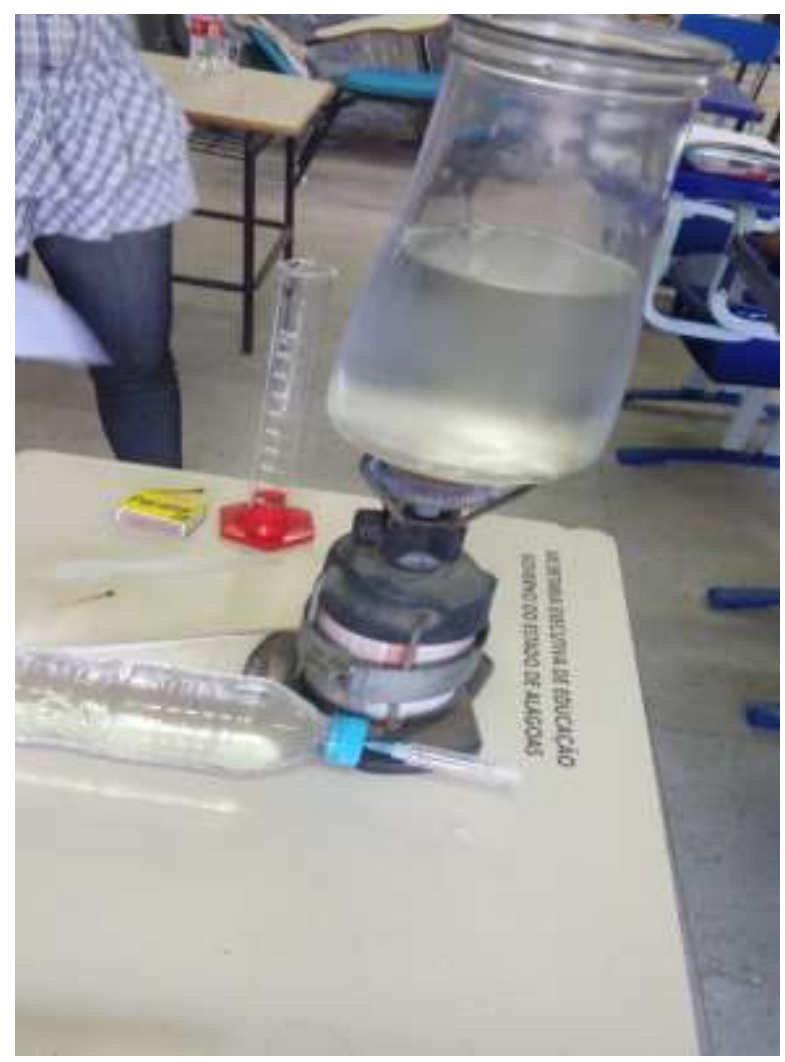

Fonte: Nelson S.Nunes, Maceió 20 junho de 2017

Em relação aos procedimentos utilizados, o trabalho foi desenvolvido nos encontros onde seguimos uma ordem pré planejada. E que foram descritos na sequência.

$1^{\circ}$ Experimento: $\mathrm{O}$ primeiro passo antes do início do experimento foi dividir os alunos em dois grupos e entregarmos aos mesmos algumas massas que estavam aferidas juntamente com um roteiro que consistia com o passo a passo para realização do experimento. Durante a realização do experimento, os alunos preencheram uma tabela[1], para poder realizar o que estava mencionado no roteiro.

Tabela 1: Tabela para os valores coletados no experimento da constante gravitacional.

\begin{tabular}{|c|c|c|c|c|c|}
\hline $\begin{array}{c}\text { Massa aferida } \\
(\mathrm{kg})\end{array}$ & Medida 1 & Medida 2 & Medida 3 & $\begin{array}{c}\text { Media } \\
<\mathrm{N}>\end{array}$ & $\begin{array}{c}\text { Gravidade } \\
\text { local }\left(\mathrm{m} / \mathrm{s}^{2}\right)\end{array}$ \\
\cline { 2 - 5 } & Força (N) & Força (N) & Força (N) & Força $<\mathrm{N}>$ & \\
\hline & & & & & \\
\hline & & & & & \\
\hline
\end{tabular}

$2^{\circ}$ Experimento: Realizamos um experimento que consistia em determinar qual a constante de uma mola. Os procedimentos que realizamos foram os mesmos do primeiro 
experimento. Novamente foi entregue um roteiro e uma tabela [2], onde deveria ser preenchida para de forma posterior se tornasse possível a construção de um gráfico.

Tabela 2: Tabela para os valores coletados no experimento da constante da mola.

\begin{tabular}{|c|c|c|c|c|c|}
\hline $\begin{array}{c}\text { Massa aferida } \\
(\mathrm{kg})\end{array}$ & Medida 1 & Medida 2 & Medida 3 & Media $<\mathrm{N}>$ & $\begin{array}{c}\text { Constante } \\
\text { da mola } \mathrm{K}\end{array}$ \\
\cline { 2 - 6 } & $\begin{array}{r}\text { Distensão } \\
\text { da mola } \Delta \mathrm{x}\end{array}$ & $\begin{array}{c}\text { Distensão } \\
\text { da mola } \Delta \mathrm{x}\end{array}$ & $\begin{array}{c}\text { Distensão } \\
\text { da mola } \Delta \mathrm{x}\end{array}$ & $\begin{array}{c}\text { Distensão da } \\
\text { mola }<\Delta \mathrm{x}>\end{array}$ & \\
\hline & & & & & \\
\hline & & & & & \\
\hline & & & & & \\
\hline
\end{tabular}

$3^{\circ}$ Experimento: $\mathrm{O}$ procedimento foi necessário que os alunos no primeiro momento colocassem a garrafa com água em um pote de gelo com sal afim de tornar essa mistura homogenia para conseguir medir um ponto de fusão abaixo de zero através dos termômetros que tinham em laboratório, observou a medida da temperatura do mistura após alguns minutos vendo o que tinha ocorrido com a água que estava presente na garrafa. No segundo momento os alunos colaram a garrafa com água em um recipiente que estava com água a $50{ }^{\circ} \mathrm{C}$ e tiveram que observar novamente o que ocorreu com a água presente na garrafa pet.

\subsection{Análises}

No primeiro experimento que realizamos foi possível observar uma determinada dificuldade dos alunos compreenderem qual o real motivo do trabalho e ainda uma complicação em relação à interpretação gráfica e modelos matemáticos que poderiam representar os mesmos.

No segundo experimento as discussões em relação à constante elástica foi mais acalorada, pois os alunos observaram a distensão da mola e relacionaram este fato a outras molas existentes no nosso dia a dia, dando origem a outros questionamentos dos alunos, tais como: "As mudanças dos valores das constantes interferem no comportamento das molas? “ "Independente da distensão, a mola sempre retornará ao seu tamanho original?”

Educação, Psicologia e Interfaces, Volume 2, Número 2, p. 77-85, Maio/Agosto, 2018. 
O ensino voltado as constantes da física, tais como gravidade local, constante da mola e coeficiente de dilatação volumétrica

"A mola de um sofá é diferente da mola de um carro por conta da constante?" "Roupas de lycra se enquadra nesse estudo?"

Com esse tipo de questionamento é possível observar que os alunos conseguiram interpretar corretamente a importância das constantes. O questionamento realizado foi respondido de forma respectiva da seguinte maneira: "Sim, a mudança de valor fará com que as molas precisem de um peso maior para possuir a mesma distensão.", "Sim, de forma ideal isso é pra acontecer, porem conforme ela vai se desgastando ao longo do tempo suas propriedades acabam se modificando, consequentemente seu tamanho original também se modifica.", "Sim, pois conforme a constante ( propriedade da mola ) ela se torna apropriada para o seu desenvolvimento correto em determinado objeto." "Sim, pois as roupas possuem propriedades elásticas tais como uma mola."

No terceiro experimento podemos observar uma maior satisfação em realizar os experimentos, onde eles conseguiam associar corretamente a relação entre as constantes e a dilatação do líquido que ocorreu durante o experimento. No geral, os alunos aceitaram bem o tipo de atividade desenvolvida e, principalmente, conseguiram construir conhecimento físico, fato demonstrado na qualidade das explicações e das indagações realizadas durante os encontros.

O trabalho foi realizado com o objetivo central de propor uma sequência de ensino e aprendizagem na área de Física, para trabalhar as constantes físicas em aulas do Ensino MédiO. Podemos relatar que os objetivos foram alcançados, afim de que os alunos pudessem construir seu conhecimento com a mínima interferência do professor e dos bolsistas PIBID, tendo em vista que a atividade 1 foi mais complicada, pois foi nela que diagnosticamos as deficiências que cada grupo apresentava, no entanto, houve uma melhora significativa na segunda atividade.

A pesquisa girou entorno do desenvolvimento do aluno de construir seu conhecimento, utilizando experimentos que demonstrassem a importância das constantes para que os mesmos começassem a identificar a sua real importância, criando assim, uma visão mais apurada do conceito produzido, o qual é algo que vai alem de um simples fator comum na física.

Portanto, a pesquisa traz no seu desenvolvimento uma contribuição para a elaboração de uma metodologia diferenciada de ensino e aprendizagem, que visa explorar mais experimentos e validando a proposta que pode ser realizada, pensada e planejada conforme o assunto que cada turma esteja estudando. Assim, é possível realizar uma 
metodologia mais atraente que a tradicional, proporcionando não só a ideia das perspectivas das constantes mais sim podendo ter validade em outra vertente.

\section{CONSIDERAÇÕES FINAIS}

Diante disso chegamos à conclusão que o mundo não é regido apenas por variáveis, mas, principalmente, regido por constantes que desempenham um papel fundamental nos modelos que descrevem a natureza dos fenômenos físicos.

A existência das constantes é importantíssima, pois é algo difícil de deter uma compreensão coesa pois à casos em que podem deixar de ser constantes e tornar variáveis, como é o caso da velocidade que é uma constante momentânea no movimento retilíneo uniforme, pois ela não varia, mas deixa de ser constante no movimento retilíneo uniformemente variado.

Há diferença também entre constantes, índices e coeficiente, onde se torna um pouco complexo compreende-las, uma característica singular entre eles dita como sendo a principal diferença, é que os índices não possuem unidades de medida no caso são grandezas adimensionais onde são representadas apenas de forma modular, por um número, diferentemente das constantes que possuem dimensão e são representadas de forma modular com direção e sentido dependendo do caso estudado.

A utilização de experimentos para demonstrar a importância das constantes no dia a dia mostrou-se muito eficaz já que durante os experimentos os próprios alunos começaram a identificar a real importância. O fato dos alunos identificarem a real relevância das constantes nos permite propor outros desdobramentos da pesquisa, explorando mais experimentos e verificando a proposta em sala de aula e no planejamento da prática docente do professor de física.

\section{REFERÊNCIAS BIBLIOGRÁFICAS}

VASQUES, Rafael. Modelagem cientifica de fenômenos físicos e o ensino de física: Física na Escola. v. 9, n. 1, p. 10-14, 2008.

LOIOLA, Jussara. Uma abordagem sócio-crítica da modelagem matemática: a perspectiva da educação matemática crítica. Alexandria Revista de Educação em Ciência e Tecnologia, v.2, n.2, p.55-68, jul. 2009.

Educação, Psicologia e Interfaces, Volume 2, Número 2, p. 77-85, Maio/Agosto, 2018. 
O ensino voltado as constantes da física, tais como gravidade local, constante da mola e coeficiente de dilatação volumétrica

\section{Credenciais dos autores}

NUNES, Nelson da Silva. Universidade Federal de Alagoas, Alagoas/Brasil. E-mail: nelsonfisica123@hotmail.com

SANTOS, José Renan Gomes dos. Professor na Universidade Federal de Alagoas, Alagoas/Brasil. E-mail: renangomesal@hotmail.com

Como citar este artigo (Formato ABNT): NUNES, Nelson da Silva; SANTOS, José Renan Gomes dos. O ensino voltado as constantes da física, tais como gravidade local, constante da mola e coeficiente de dilatação volumétrica. Educação, Psicologia e Interfaces, v. 2, n. 2, p. 77-85, 2018. DOI: https://doi.org/10.37444/issn-25945343.v2i2.61

Recebido: $10 / 02 / 2018$.

Aceito: 26/06/2018. 